\title{
New Centromere Autoantigens Identified in Systemic Sclerosis Using Centromere Protein Microarrays
}

\author{
Guang Song, Chaojun Hu, Heng Zhu, Li Wang, Fengchun Zhang, Yongzhe Li, and Lin Wu
}

ABSTRACT. Objective. To identify novel centromere protein (CENP) targets of anticentromere antibodies (ACA), and to investigate their association with clinical manifestations of systemic sclerosis (SSc).

Methods. A CENP-focused protein microarray was fabricated by spotting 14 purified CENP. These microarrays were individually incubated with 35 ACA-positive SSc sera and 20 ACA-negative healthy control samples. Newly identified CENP autoantigens with high sensitivities were selected for validation and characterization.

Results. Statistical analysis revealed 11 CENP are potential target antigens of ACA in patients with SSc. Of them, 5 [CENP-P, CENP-Q, CENP-M (isoform I), CENP-J, and CENP-T] are novel, among which CENP-P and CENP-Q showed high sensitivities in ACA-positive SSc sera of $34.3 \%$ and $28.6 \%$, respectively. Subsequently, 186 SSc sera (35 ACA-positives and 151 negatives), 69 ACA-positive sera from other various autoimmune diseases (primary Sjögren syndrome, systemic lupus erythematosus, rheumatoid arthritis, and primary biliary cirrhosis), and 31 healthy sera were assayed for the presence of anti-CENP-P and -Q autoantibodies by ELISA followed by Western blotting analysis. CENP-P and -Q autoantibodies were detected in ACA-positive sera of various disease groups; among them, SSc showed the highest detection rate. Anti-CENP-P was also found in 9 of the 151 ACA-negative sera. Analyses of the correlation with clinical information showed anti-CENP-P-positive patients had higher levels of $\mathrm{IgG}$, IgA, and erythrocyte sedimentation rate among the ACA-positive cohort and were more vulnerable to renal disease in the ACA-negative patients with SSc. Regardless of ACA status, anti-CENP-P or Q-negative patients seem to be predominantly affected by interstitial lung disease.

Conclusion. CENP-P and CENP-Q were identified as novel ACA autoantigens by CENP microarray assays followed by validation of ELISA and Western blotting. Both of them have prognostic utility for interstitial lung disease. CENP-P was associated with renal disease in an ACA-negative cohort. (First Release Feb 15 2013; J Rheumatol 2013;40:461-8; doi:10.3899/jrheum.120264)

Key Indexing Terms:

ANTICENTROMERE ANTIBODY

SYSTEMIC SCLEROSIS

From the Beijing Institute of Genomics, Chinese Academy of Sciences, Beijing; Department of Rheumatology and Clinical Immunology, Peking Union Medical College Hospital, Peking Union Medical College, Chinese Academy of Medical Sciences, Beijing, P.R. China; The Center for High-Throughput Biology, Johns Hopkins University School of Medicine, Baltimore, Maryland, USA; and the Graduate University of Chinese Academy of Sciences, Beijing, P.R. China.

Supported in part by grants from the Ministry of Science and Technology of the People's Republic of China (to LW: 2006AA02A311 and 2009CB522204), the National Science Technology Pillar Program in the 11th Five-Year Plan (to YL: 2008BAI59B02 and 2008BAI59B03), the National Natural Science Foundation of China (to YL: 30471617 , 30640084, 30872331, and 81072486), and the US National Institutes of Health (to HZ: R44 CA125807, U24 CA160036, U54 RR020839).

G. Song, PhD; L. Wu, PhD, Professor, Beijing Institute of Genomics, Chinese Academy of Sciences, Graduate University of Chinese Academy of Sciences; C. Hu, MD; L. Wang, MD; F. Zhang, MD, Professor; Y. Li, $M D$, Professor, Department of Rheumatology and Clinical Immunology, Peking Union Medical College Hospital, Peking Union Medical College, Chinese Academy of Medical Sciences; H. Zhu, PhD, Associate Professor, The Center for High-Throughput Biology, Johns Hopkins University School of Medicine.

G. Song and C. Hu contributed equally to this work.

Address correspondence to Dr. L. Wu, Beijing Institute of Genomics, Chinese Academy of Sciences, Beijing 100029, People's Republic of China.E-mail:wul@big.ac.cn,yongzhelipumch@yahoo.com.cn.

Accepted for publication December 27, 2012.

\section{PROTEIN MICROARRAY BIOMARKERS}

Anticentromere antibodies (ACA) were first described in 1980 by incubation of human epithelial cell line 2 (HEp-2) cell substrates with the sera of patients with CREST syndrome (calcinosis, Raynaud phenomenon, esophageal dysmotility, sclerodactyly, and telangiectasias) using indirect immunofluorescence (IIF) ${ }^{1}$. The IIF pattern normally shows discrete speckled nucleoplasmic staining in HEp-2 cells at the interphase and distinct centromeric dots that are visible for each chromosome pair in the metaphase plate. ACA have been repeatedly demonstrated as useful biomarkers in the diagnosis of systemic sclerosis (SSc), where their sensitivity is about $20 \%-35 \%$. ACA are closely associated with CREST syndrome, a limited form of SSc, and can also serve as predictors of a more benign and protracted course ${ }^{2}$. In addition, ACA are thought to be associated with pulmonary arterial hypertension ${ }^{3,4}$ and cardiac conduction blocks 5 .

Other than in SSc, ACA have been found in several other autoimmune diseases and cancers such as primary biliary cirrhosis (PBC; $18 \%-26.1 \%)^{6,7,8}$, primary Sjögren syndrome $(\mathrm{pSS} ; 3.7 \%)^{9}$, systemic lupus erythematosus 
$(\mathrm{SLE} ; 1.9 \%-11 \%)^{10,11,12}$, rheumatoid arthritis $(\mathrm{RA} ; 3 \%)^{11}$, and breast cancer ${ }^{13}$.

To date, several centromere proteins (CENP) have been identified as ACA autoantigens, among which CENP-B, CENP-A, and CENP-C are predominant. Immunostrips and/or ELISA assays using recombinant CENP-A and CENP-B have been used in some clinics for detection of ACA. Other known target antigens of ACA include CENP-D ${ }^{14,15}$, CENP-E $^{16}$, and CENP-O $^{17}$ discovered in

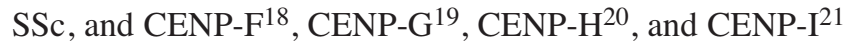
in other diseases. However, a recent study tested 95 sera with ACA, and found none reacted to CENP-H, -I, -K, $-\mathrm{L},-\mathrm{M},-\mathrm{N}$, and $-\mathrm{U}$, and only 1 reacted weakly to CENP-T ${ }^{22}$. Additionally, autoantibodies against several CENP have been associated with clinical manifestations, for example, the level of anti-CENP-B antibodies correlated with a less extensive skin involvement in $\mathrm{SSc}^{23}$, and pSS patients with anti-CENP-H antibodies had a lower frequency of rheumatoid factor than those without ${ }^{20}$. Additionally, Gelber, et al found that the dual presence of anti-CENP-B and -C was most frequently seen in SSc, while anti-CENP-C alone was predominantly found in patients with $\mathrm{pSS}$, and further suggested that obtaining antibodies to specific centromere antigens was diagnostically useful ${ }^{24}$.

During eukaryotic cell division at the centromere locus, a multiprotein complex known as the kinetochore is assembled, which involves many CENP such as $-\mathrm{A},-\mathrm{C},-\mathrm{H},-\mathrm{M},-\mathrm{N},-\mathrm{T}$, and MLF1IP/CENP-U in the CENP-A-NAC complex, and -I, $-\mathrm{K},-\mathrm{L},-\mathrm{O},-\mathrm{P},-\mathrm{Q},-\mathrm{R}$, and $-\mathrm{S}$ in the CENP-A-CAD complex that interacts with the CENP-A-NAC complex ${ }^{25}$. However, whether other CENP have autoimmune activity has not been comprehensively surveyed.

To address this question, we first used a CENP-focused protein microarray composed of $14 \mathrm{CENP}$ (A, B, C, H, I, J, $\mathrm{K}, \mathrm{L}, \mathrm{M}, \mathrm{N}, \mathrm{O}, \mathrm{P}, \mathrm{Q}, \mathrm{T})$ to profile ACA-positive SSc sera judged by IIF and/or immunostrip as in clinical practice. The new candidate CENP autoantigens identified by the microarrays were further tested by ELISA and Western blotting (WB) to validate the findings and to analyze their association with clinical manifestations.

\section{MATERIALS AND METHODS}

Serum samples. A total of 186 Chinese patients (171 women, age $44.6 \pm$ $12.3 \mathrm{yrs}$ ) with SSc were enrolled. All of them fulfilled the American College of Rheumatology (ACR) classification criteria for $\mathrm{SSc}^{26}$, and were subcategorized into limited SSc and diffuse SSc according to the classification system proposed by LeRoy, et $a^{27}$. Also included were sera from 31 healthy volunteers ( 12 women, age $38.3 \pm 11.9 \mathrm{yrs}$ ) and 69 ACA-positive patients affected by various autoimmune diseases including $18 \mathrm{pSS}$ (all women, age $54.7 \pm 10.2 \mathrm{yrs}), 18 \mathrm{PBC}$ ( 17 women, age $58.7 \pm 11.4 \mathrm{yrs}$ ), 20 SLE (all women, age $44.7 \pm 15.7 \mathrm{yrs}$ ), and $13 \mathrm{RA}$ (11 women, age $59.4 \pm$ $13.0 \mathrm{yrs})$. PBC was diagnosed according to the criteria from the American Association for the Study of Liver Diseases ${ }^{28}$; pSS fulfilled the AmericanEuropean Consensus Group Classification criteria ${ }^{29}$; SLE and RA fulfilled the corresponding classification criteria from the $\mathrm{ACR}^{30,31}$.

All samples were collected between January 2008 and December 2009 at Peking Union Medical College Hospital. Informed consent in writing was obtained from each participant. Our study was conducted with the approval of the Ethics Committee of the Peking Union Medical College Hospital.

Clinical measurements. Clinical assessment of organ manifestation in SSc was performed according to the report from the European League Against Rheumatism ${ }^{32}$. In short, the definitions of systemic involvement are as follows: cardiac involvement (arrhythmia and conductive block as revealed by electrocardiogram, systolic/diastolic dysfunction, pericardial effusion, and pulmonary arterial hypertension, estimated pulmonary arterial systolic pressure $>40 \mathrm{~mm} \mathrm{Hg}$, by echocardiogram); lung involvement [bilateral basilar velcro sounds by auscultation, evidence of interstitial lung disease (ILD) as demonstrated by chest radiograph, high-resolution computerized tomography (HRCT), and pulmonary function test (total lung capacity < $70 \%$ of predicted value, DLCO $<70 \%$ of predicted value)]; renal involvement (symptoms of nephrogenic peripheral edema, decreased daily urine output, and renal crisis diagnosed by clinician); and gastrointestinal manifestations (dysphagia, sour reflux, vomiting, diarrhea, constipation, and abdominal distension and decreased bowel sounds by auscultation).

Additionally, we analyzed the levels of IgG and IgA as well as erythrocyte sedimentation rate (ESR) for each patient with SSc. These are considered important indicators of autoimmune disease activity.

Detection of ACA. All samples were subjected to IIF and immunoblot testing using commercial kits according to the manufacturer's protocol (EuroImmun Medizinische Labordiagnostika AG). For IIF on HEp-2 cells, serum samples were considered ACA-positive at titers $\geq 160$, and all negative sera showed no staining of ACA-specific pattern at titers of 1:40 and 1:80. The immunoblot strips contain CENP-A, CENP-B, and other SSc-related autoantigens including Scl-70, RNA polymerase, U3-RNP, and $\mathrm{Th} / \mathrm{To}$. The serum samples that were positive by IIF, CENP-A, or CENP-B were determined as ACA-positive. In total, 35 from the $186 \mathrm{SSc}$ sera samples were identified as ACA-positive. Of them, 30 were positive by all 3 measures, 2 by both CENP-A and CENP-B, 1 by both CENP-B and IIF, and 1 each by CENP-B or IIF only.

Preparation of CENP and construction of CENP microarrays. A total of 16 recombinant proteins for 14 CENP (CENP-M and CENP-N have 2 isoforms each) were expressed and purified as glutathione S-transferase (GST) fusions in yeast as described ${ }^{33}$. All the purified CENP, together with controls (printing buffer, GST, nucleoprotein of influenza, and human IgG), were printed in triplicate within 12 identical probe areas on each OPEpoxySlide (CapitalBio Corp.). The CENP microarrays were stored at $4^{\circ} \mathrm{C}$ under vacuum until used.

Serum profiling with CENP microarrays. Thirty-five ACA-positive SSc sera and 20 ACA-negative healthy sera samples were individually incubated with the CENP microarrays, and the bound antibodies were detected following the procedure described ${ }^{4}$. We set the cutoff at $3 \mathrm{SD}$ above the average signal intensity from healthy sera. The CENP were considered positive only when at least 2 of the triplicates were positive.

The 96-well plate was coated at $4^{\circ} \mathrm{C}$ overnight with recombinant CENP-P and CENP-Q proteins at $200 \mathrm{ng}$ and $50 \mathrm{ng} / 100 \mu \mathrm{l} / \mathrm{well}$, respectively. The ELISA was carried out as described ${ }^{34}$. The optical density (OD) at $450 \mathrm{~nm}$ was measured and the mean OD of the duplicate wells for each serum sample was used for data analysis. Because the CENP proteins are GST-fused, ELISA using equal molar amounts of GST protein were also conducted in parallel. The GST readings were subtracted from the readings of CENP. Cutoff values were set at $5 \mathrm{SD}$ above the mean value from healthy samples.

Western blot analysis. Five hundred nanograms of recombinant GST-tagged CENP-P or -Q were resolved by $12 \%$ sodium dodecyl sulfate-polyacrylamide gel electrophoresis. The separated proteins were transferred onto PVDF membranes (Millipore). After being blocked with 5\% nonfat milk, the membranes were incubated with sera (1:500), followed by horseradish peroxidase-conjugated anti-human IgG. The immunoreactive bands were visualized by chemiluminescence (Beijing Applygen Ltd. Co.). Statistical analysis. P values were calculated with Pearson's chi-square or 
Fisher's exact test using the R programming language and the Student $\mathrm{t}$ test if necessary. $\mathrm{P}$ values $<0.05$ were considered statistically significant.

\section{RESULTS}

Construction of CENP protein microarray. Sixteen open reading frames $(\mathrm{ORF})$ representing $14 \mathrm{CENP}(\mathrm{A}, \mathrm{B}, \mathrm{C}, \mathrm{H}, \mathrm{I}$, J, K, L, M, N, O, P, Q, T; 2 ORF for both $\mathrm{M}$ and N) cloned into the pEGHA vector (supplementary data for $16 \mathrm{ORF}$ available from the author upon request) were expressed and purified as GST fusions in yeast. Subsequently, each CENP, together with negative and positive controls (Figure 1A), was printed in triplicate within 12 identical blocks on a single slide to produce the CENP microarray. To evaluate the quality of the microarrays, an anti-GST monoclonal antibody was used to visualize and quantify the CENP immobilized on the slides (Figure 1B). All CENP on the microarray produced GST signals significantly above background and each feature showed highly reproducible signals with pairwise correlation coefficients of 0.999 , indicating that this microarray is of high quality (Figure 1, B-C).

Identification of new CENP antigens for ACA by protein microarray. To determine whether additional CENP could be recognized by ACA, 35 ACA-positive SSc sera were individually incubated with the CENP microarrays (Figure 1, D-I). As a comparison, a cohort of 20 ACA-negative healthy subjects was also assayed in the same fashion. Using the mean value plus 3 SD of the healthy subjects as a cutoff, 11 of the 16 CENP showed various positive rates across the panel of ACA-positive SSc sera (Figure 2; supplementary data available from the author upon request), and none of the healthy sera were scored as positive. As expected, the 3 prominent antigens, CENP-B, CENP-A, and CENP-C, showed the highest positive rates of $97.1 \%(34 / 35), 77.1 \%$

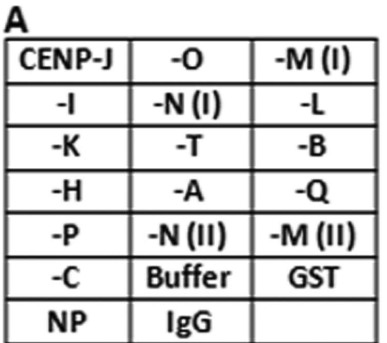

Location of all probes

D

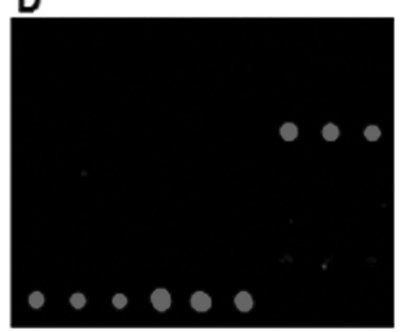

SSc092

G

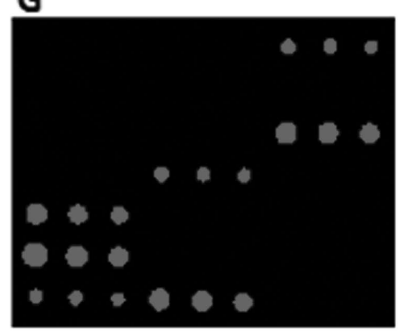

SSc167
B

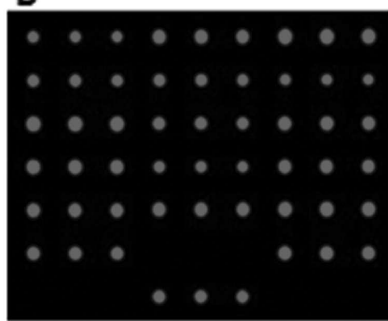

Anti-GST mAb

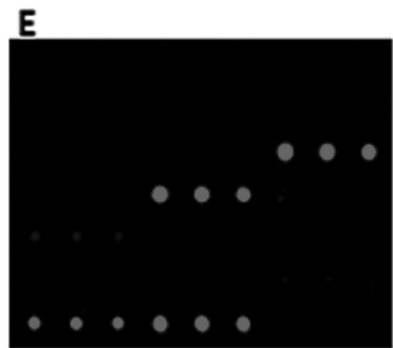

$\mathrm{SSc028}$

H

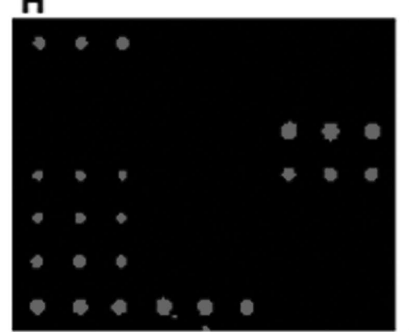

SSc070
C

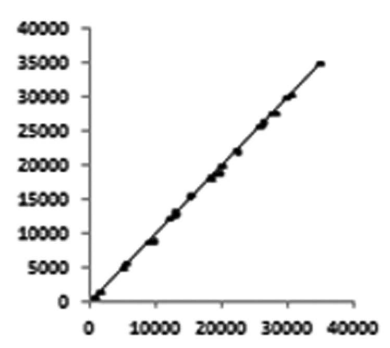

$\mathbf{F}$

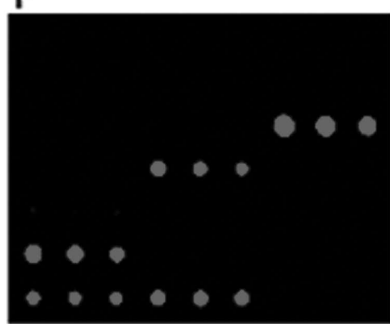

SSc118

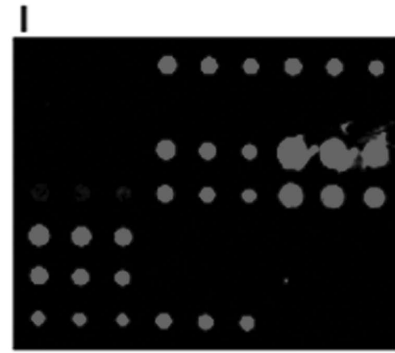

SSc100

Figure 1. Profiling systemic sclerosis (SSc) sera with centromere protein (CENP) microarrays. A. Layout of CENP microarray. Each probe was printed in triplicate. $\mathrm{M}(\mathrm{I}), \mathrm{M}(\mathrm{II})$, and $\mathrm{N}(\mathrm{I}), \mathrm{N}(\mathrm{II})$ stand for isoform I and isoform II of CENP-M and CENP-N, respectively. NP (nucleoprotein of influenza), IgG, GST, and buffer are various controls. B. Scanned image of the CENP microarray probed with anti-GST antibody. The 2 negative controls (NP and buffer) did not show any signals, while all the other proteins, including IgG, showed strong and rather even signals. C. Reproducibility of triplicate protein probes detected by anti-GST antibody shown in B. The signal intensities of any 2 of the triplicate spots are plotted, and the correlation coefficiency is 0.9991 , indicating good quality. D-I. Representative images of the CENP microarrays probed with anticentromere antibody-positive SSc sera. mAb: monoclonal antibody. 


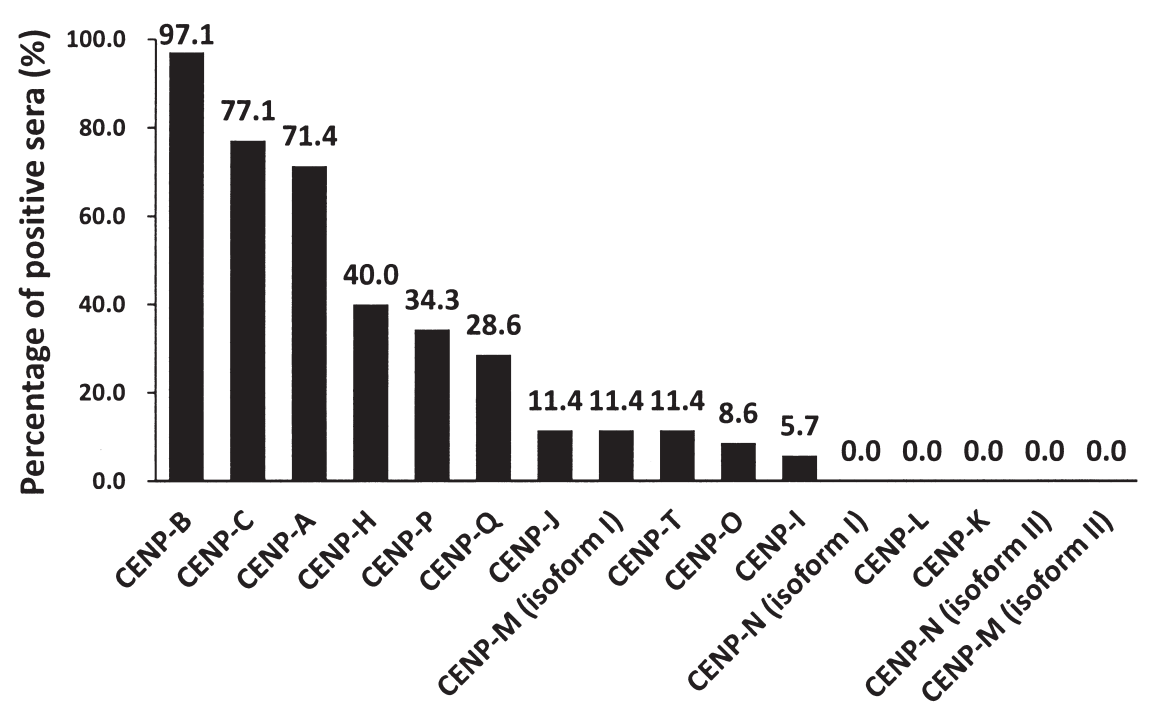

Figure 2. Percentage of anticentromere antibody (ACA)-positive systemic sclerosis (SSc) sera positive to each centromere protein (CENP) in the CENP microarray analysis. Thirty-five ACA-positive SSc sera samples were incubated with CENP microarrays, and the percentage of the positive sera for each CENP is plotted.

(27/35), and $71.4 \%$ (25/35), respectively, while 3 other known antigens, CENP-H, CENP-O, and CENP-I, could be recognized by $40 \%(14 / 35), 8.6 \%$ (3/35), and $5.7 \%(2 / 35)$ of the samples. Importantly, we were able to identify 5 new candidate ACA autoantigens [CENP-P, -Q, -J, -M (isoform I), and $-\mathrm{T}$ ] that showed positive rates of $34.3 \%$ (12/35), $28.6 \%$ (10/35), $11.4 \%$ (4/35), $11.4 \%$ (4/35), and $11.4 \%$ (4/35), respectively.

Validation of CENP-P and CENP-Q autoantibodies by ELISA and Western blot. To validate the newly identified autoantigens, CENP-P and -Q were selected for further analysis because of their relatively high sensitivities. Sera from 186 patients with SSc (35 ACA-positive and 151 ACA-negative) and 31 healthy individuals (ACA-negative) were used to conduct the ELISA assay (supplementary data available from the author upon request). In total, 31 and 12 SSc sera were positive for CENP-P and -Q, respectively (supplementary data available from the author upon request). To validate them, all of these positives were subjected to WB using recombinant CENP-P and -Q proteins. We also included GST protein because CENP-P and -Q proteins were GST-tagged. No significant signals were observed for the GST protein $(\sim 26 \mathrm{kDa})$ for all samples (Figure 3). Twenty-three and 11 sera gave expected immunoreactive bands at 58-62 kDa for CENP-P and CENP-Q, respectively, and were considered true positives (Figure 3, Table 1).

For ACA-positive SSc sera, CENP-P and -Q showed a respective positive rate of $40 \%(14 / 35)$ and $31.4 \%$ (11/35), slightly higher than the microarray results. Interestingly, of the 151 ACA-negative SSc sera, 9 were CENP-P positive.

It is well known that ACA are not unique to SSc; many patients with other autoimmune diseases have them, although with lower frequency $6,7,8,9,10,11,12$. To comprehensively characterize the behavior of CENP-P and -Q autoantibodies in these autoimmune diseases, we also included ACA-positive sera from 18 patients with pSS, 18 with PBC, 20 with SLE, and 13 with RA (Figure 3, Table 1). Not surprisingly, CENP-P and -Q autoantibodies were detected in $\mathrm{pSS}$, SLE, RA, and/or PBC at various rates.

Association of CENP-P and CENP-Q autoantibodies with clinical manifestations and laboratory features in SSc. To determine whether the newly identified autoantigens, CENP-P and -Q, have any diagnostic value for SSc, we performed additional statistical tests (chi-square/Fisher's exact test and T-test) to examine potential associations between the CENP-P or -Q autoantibodies and clinical manifestations and/or other laboratory features.

The analysis for anti-CENP-P is summarized in Table 2. For ACA-positive SSc sera, the serum levels of $\operatorname{IgG}$ and $\operatorname{IgA}$ in the patients with anti-CENP-P were significantly higher than those of patients negative for anti-CENP-P $(p<0.05)$. Meanwhile, ESR in patients who were anti-CENP-P-positive was also significantly higher than in patients who were anti-CENP-P-negative ( $\mathrm{p}=0.0075)$. For ACA-negative SSc sera, the percentage of patients with renal involvement was significantly higher in anti-CENP-P-positive patients than in anti-CENP-P-negative patients $(\mathrm{p}=0.042)$. Additionally, regardless of ACA status, patients with SSc who were positive for anti-CENP-P showed significant association with renal involvement $(\mathrm{p}=0.0418)$ but were less affected by ILD ( $p=0.0218)$. As well, the mean age of these patients seems to be higher than that of patients without anti-CENP-P (mean age \pm SD $49.9 \pm 13.5$ vs $43.8 \pm 12$ yrs; $p=0.0267$ ). Further, anti-CENP-P was prevalent in both anti-CENP-A and $-\mathrm{B}$-positive cohorts $(\mathrm{p}<0.0001)$, although there were

Personal non-commercial use only. The Journal of Rheumatology Copyright $\odot$ 2013. All rights reserved. 

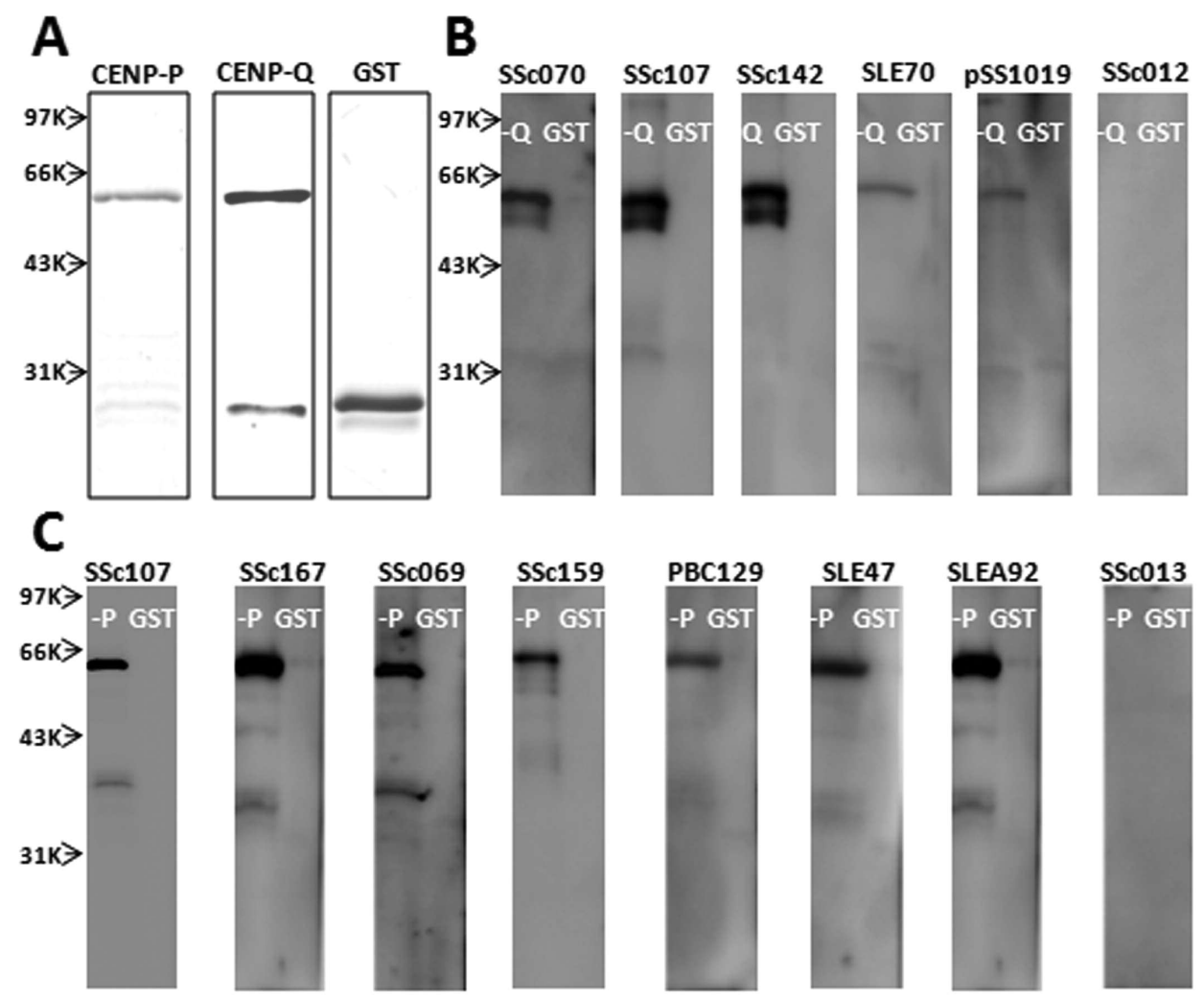

Figure 3. Validation of centromere protein (CENP)-P and CENP-Q autoantibodies by Western blotting. A. Recombinant GST-tagged CENP-P, CENP-Q, and GST proteins in SDS-PAGE. B. Blotted recombinant CENP-Q $(58.6 \mathrm{kDa})$ and GST $(28 \mathrm{kDa})$ proteins were probed with representative anti-CENP-Q ELISA-positive (SSc070, SSc107, SSc142, SLE70, and pSS1019) and -negative (SSc012) sera. C. Blotted recombinant CENP-P (61.2 $\mathrm{kDa}$ ) and GST proteins were probed with representative anti-CENP-P ELISA-positive (SSc107, SSc167, SSc069, SSc159, PBC129, SLE47, and SLEA92) and -negative (SSc013) sera. GST: glutathione S-transferase; SSc: systemic sclerosis; SLE: systemic lupus erythematosus; PBC: primary biliary cirrhosis.

Table 1. Positive sera against centromere protein (CENP)-P and -Q in systemic sclerosis ( $\mathrm{SSc}$ ) and other cohorts. Positives to CENP-P/-Q were determined by ELISA and Western blot methods. P value was calculated using Pearson's chi-square or Fisher's exact test. P values $<0.05$ are shown in bold type, indicating statistically significant differences compared to anticentromere antibody (ACA)-positive.

\begin{tabular}{lccc}
\hline Cohort & Cases & $\begin{array}{c}\text { Anti-CENP-P }(+) \\
\text { No. }(\%, \mathrm{p} \text { value })\end{array}$ & $\begin{array}{c}\text { Anti-CENP-Q }(+) \\
\text { No. }(\%, \mathrm{p} \text { value })\end{array}$ \\
\hline SSc & & & \\
$\quad$ ACA+ & 35 & $14(40)$ & $11(31.4)$ \\
ACA- & 151 & $9(6,<\mathbf{0 . 0 0 0 1})$ & $0(0,<\mathbf{0 . 0 0 0 1})$ \\
pSS (ACA+) & 18 & $1(5.6, \mathbf{0 . 0 2 0 7})$ & $2(11.1,0.1771)$ \\
SLE (ACA+) & 20 & $3(15,0.1038)$ & $5(25,0.7608)$ \\
RA (ACA+) & 13 & $2(15.4,0.1703)$ & $0(0, \mathbf{0 . 0 2 2 8})$ \\
PBC (ACA+) & 18 & $5(27.8,0.5644)$ & $1(5.6, \mathbf{0 . 0 4 1 2})$ \\
Healthy (ACA-) & 31 & $0(0, \mathbf{0 . 0 0 0 2})$ & $0(0, \mathbf{0 . 0 0 2 0})$ \\
\hline
\end{tabular}

SLE: systemic lupus erythematosus; pSS: primary Sjögren syndrome; RA: rheumatoid arthritis; PBC: primary biliary cirrhosis.

still $39.1 \%$ and $43.5 \%$ of sera samples with anti-CENP-P that did not have anti-CENP-B and -A, respectively.

As for anti-CENP-Q (Table 3), its presence did not show significant association with any clinical manifestations and laboratory features in the ACA-positive cohort. However, regardless of ACA status, the presence of anti-CENP-Q was strongly associated with limited SSc and showed strong association with a lower prevalence of ILD ( $p=0.003)$. In relation to other $\mathrm{SSc}$ autoantibodies, all the sera with anti-CENP-Q were also positive for both anti-CENP-B and -A, but anti-CENP-Q was more prevalent in the cohort negative for anti-Scl70 ( $\mathrm{p}=0.0244)$, an autoantibody associated with diffuse SSc.

\section{DISCUSSION}

Protein microarray technology has emerged as a high-throughput method for autoantigen identification, and has been successfully used for profiling autoantibodies in different types of diseases ${ }^{34,35,36}$. For patients with SSc, ACA have been shown to be useful diagnosis biomarkers for phenotypic subsets, as well as prognosis markers ${ }^{37}$. We fabricated a CENP-focused protein microarray to comprehensively survey their autoimmunity status in patients with SSc. In addition to the recovery of 6 known ACA antigens, 5 CENP were identified as potential novel autoantigens in SSc. Validation results from ELISA and Western blotting demonstrated that both CENP-P and -Q are actual centromere 
Table 2. Association analysis between anti-CENP-P antibody in systemic sclerosis (SSc) sera and clinical manifestations and laboratory features. Bold type indicates p values $<0.05$, considered statistically significant. Data are percentages unless indicated otherwise.

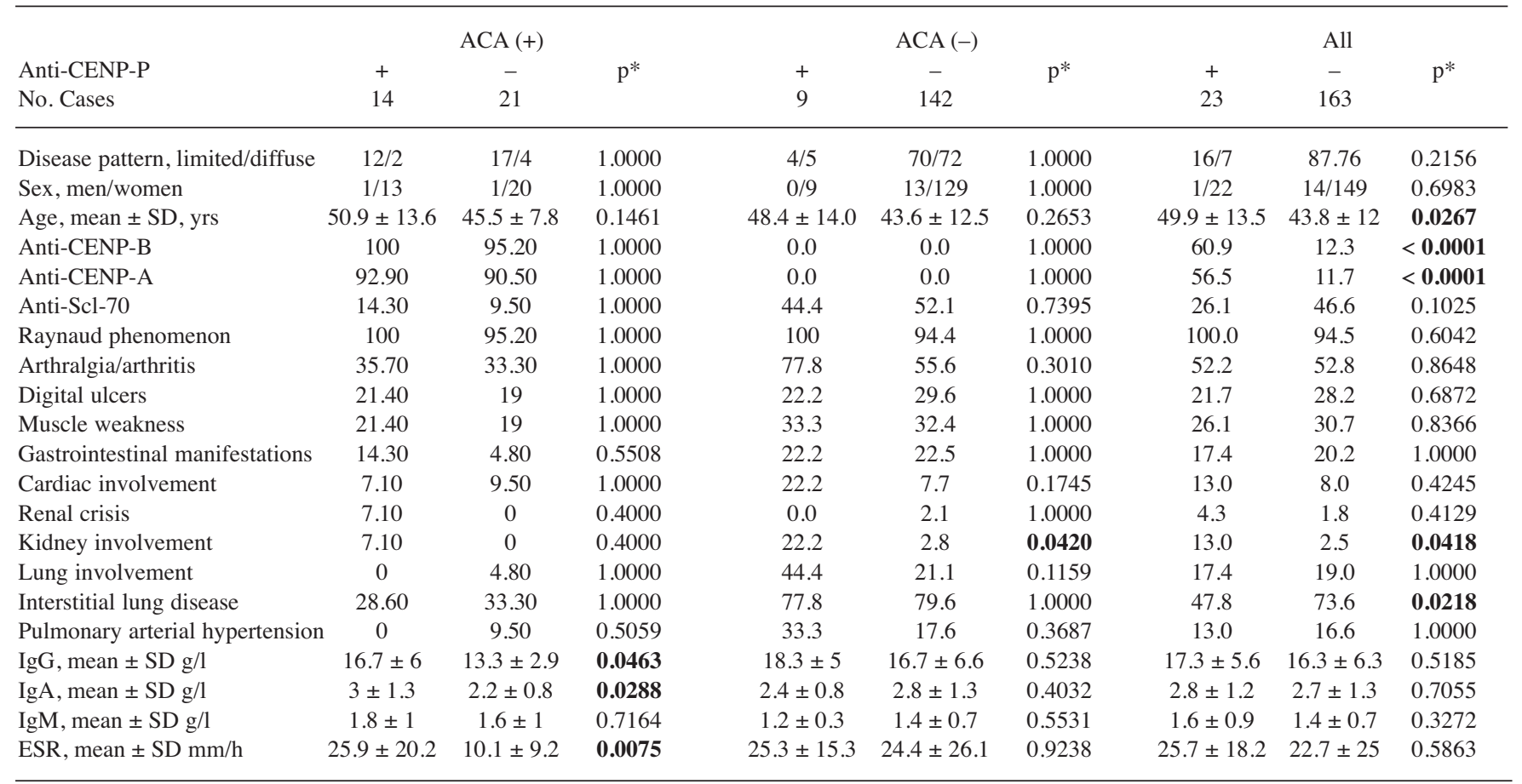

* P values calculated using Pearson's chi-square or Fisher's exact test and 2-tailed T-test if necessary. CENP: centromere protein; ESR: erythrocyte sedimentation rate; ACA: anticentromere antibodies.

Table 3. Association analysis between anti-CENP-Q antibody in systemic sclerosis sera samples and clinical manifestations and laboratory features. Bold type indicates $\mathrm{p}$ values $<0.05$, which are statistically significant. Data are percentages unless otherwise indicated.

\begin{tabular}{lcccccc}
\hline & \multicolumn{7}{c}{ ACA $(+)$} & & & All & \\
Anti-CENP-Q & + & - & $\mathrm{p}^{*}$ & + & - & $\mathrm{p}^{*}$ \\
No. Cases & 11 & 24 & & 11 & 175 & \\
\hline Disease pattern, limited/diffuse & $10 / 1$ & $19 / 5$ & 0.6400 & $10 / 1$ & $93 / 82$ & $\mathbf{0 . 0 2 4 3}$ \\
Sex, men/women & $1 / 10$ & $1 / 23$ & 0.5361 & $1 / 10$ & $14 / 161$ & 1.0000 \\
Age, mean \pm SD, yrs & $49.2 \pm 12.7$ & $46.9 \pm 9.8$ & 0.5674 & $49.2 \pm 12.7$ & $44.3 \pm 12.3$ & 0.2040 \\
Anti-CENP-B & 100 & 95.8 & 1.0000 & 100 & 13.1 & $<\mathbf{0 . 0 0 0 1}$ \\
Anti-CENP-A & 100 & 87.5 & 0.5361 & 100 & 12.0 & $<\mathbf{0 . 0 0 0 1}$ \\
Anti-Scl-70 & 9.1 & 12.5 & 1.0000 & 9.1 & 46.3 & $\mathbf{0 . 0 2 4 4}$ \\
Raynaud phenomenon & 90.9 & 100 & 0.3143 & 90.9 & 95.4 & 0.4295 \\
Arthralgia/arthritis & 36.4 & 33.3 & 1.0000 & 36.4 & 53.7 & 0.4198 \\
Digital ulcers & 18.2 & 20.8 & 1.0000 & 18.2 & 28.0 & 0.7300 \\
Muscle weakness & 36.4 & 12.5 & 0.1715 & 36.4 & 29.7 & 0.7365 \\
GI manifestations & 18.2 & 4.2 & 0.2269 & 18.2 & 20.0 & 1.0000 \\
Cardiac involvement & 9.1 & 8.3 & 1.0000 & 9.1 & 8.6 & 1.0000 \\
Renal crisis & 9.1 & 0 & 0.3143 & 9.1 & 1.7 & 0.2180 \\
Kidney involvement & 9.1 & 0 & 0.3143 & 9.1 & 3.4 & 0.3521 \\
Lung involvement & 0 & 4.2 & 1.0000 & 0.0 & 20.0 & 0.1290 \\
Interstitial lung disease & 27.3 & 33.3 & 1.0000 & 27.3 & 73.1 & $\mathbf{0 . 0 0 3 0}$ \\
PAH & 0 & 8.3 & 1.0000 & 0.0 & 17.1 & 0.2165 \\
IgG, mean \pm SD g/l & $15.8 \pm 6.2$ & $14.3 \pm 4$ & 0.4191 & $15.8 \pm 6.2$ & $16.5 \pm 6.3$ & 0.7288 \\
IgA, mean \pm SD g/l & $2.8 \pm 0.9$ & $2.4 \pm 1.3$ & 0.4034 & $2.8 \pm 0.9$ & $2.7 \pm 1.3$ & 0.8354 \\
IgM, mean \pm SD g/l & $1.7 \pm 0.6$ & $1.7 \pm 1.2$ & 0.9001 & $1.7 \pm 0.6$ & $1.4 \pm 0.8$ & 0.3017 \\
ESR, mean \pm SD mm/h & $17.6 \pm 15.6$ & $16.4 \pm 17.4$ & 0.8451 & $17.6 \pm 15.6$ & $23.4 \pm 24.7$ & 0.4442 \\
\hline
\end{tabular}

* P values calculated using Pearson's chi-square or Fisher's exact test and 2-tailed T-test if necessary. CENP: centromere protein; ACA: anticentromere antibodies; GI: gastrointestinal; PAH: pulmonary arterial hypertension; ESR: erythrocyte sedimentation rate. 
autoantigens for ACA. In addition, anti-CENP-P autoantibodies were also present in ACA-negative sera (6.0\%).

In theory, a serum sample that can recognize a CENP should be able to stain the kinetochore and show an ACA-positive pattern in the IIF assay. However, we found that quite a few sera samples found to be ACA-negative by IIF were actually anti-CENP-B and/or -A positive by both commercial immunostrip assay and centromere protein microarray. Similarly, anti-CENP-P was found in IIF-negative sera. This discrepancy may suggest that either the IIF method is not sensitive enough, requiring higher antibody titer, or the CENP-P protein/epitopes are buried in the complex that blocks the access of their autoantibodies. On the other hand, 1 serum sample determined by IIF to be ACA-positive failed to recognize any of the 14 CENP in our microarray analysis as well as CENP-A and CENP-B by commercial immunostrips (supplementary data available from the author upon request), suggesting there might be additional autoantigens in the kinetochore yet to be discovered.

Historically, the presence of an ACA-associated staining pattern by IIF was a strong serological indicator for limited SSc or CREST syndrome ${ }^{4}$, as well as a predictor of a more benign and protracted course for $\mathrm{SSc}^{2}$. It was also reported that ACA are much less frequently found in patients with ILD $^{2}$. Similar results were obtained by surveying the 186 SSc serum samples in our study. Among the serologically ACA-positive patients, $82.9 \%$ (29/35) displayed a limited cutaneous form of SSc, and only $31.4 \%$ (11/35) of them were affected by ILD; whereas as many as $79.5 \%(120 / 151)$ of ACA-negative patients were diagnosed with the same diseases. The significantly reduced risk for ILD was also found in patients with anti-CENP-Q autoantibodies ( $\mathrm{p}<$ 0.01 ) in our study.

Moreover, we discovered that patients with anti-CENP-P autoantibodies were more vulnerable to renal diseases in the ACA-negative cohort $(\mathrm{p}=0.042)$, and even in the whole SSc cohort $(p=0.0418)$. The associations of anti-CENP-P antibodies with renal involvement are different from traditional clinical features associated with $\mathrm{ACA}^{2}$. This might suggest that anti-CENP-P antibody has unique clinical associations in SSc, especially in the setting of the ACA (negative) by traditional methods in the clinic. In addition, we found that anti-CENP-P did not coexist with anti-RNA polymerase, anti-U3-RNP, or anti-Th/To (data not shown), the autoantibodies for assessment of organ involvement in SSc. Therefore, anti-CENP-P may be a useful biomarker in clinical diagnosis of SSc.

Studies show that the prevalence of SSc-specific autoantibodies is different among various ethnic groups ${ }^{38}$. ACA appear to be much less frequent in some ethnic groups such as Thais $(\sim 2 \%)$, African Americans $(\sim 0 \%)$, and South African blacks $(\sim 0 \%)^{2,39,40,41}$, but more frequent in whites $(17 \%)^{42}$, Danes $(34 \%)^{43}$, and white adults in the United States $(27 \%)^{44}$. In our study, the samples were all from
Chinese subjects. Therefore the usefulness of the newly identified CENP autoantigens in diagnosis of SSc for other groups needs further research.

In addition to CENP-P and -Q, 3 CENP antigens [CENP-J, -M (isoform I), and -T] were identified by microarray technology as new candidate ACA autoantigens, albeit at a lower occurrence. However, whether the autoantibodies against these 3 CENP are associated with specific clinical manifestations or laboratory features needs further analysis with larger cohorts. In our microarray, we included 2 isoforms for CENP-M, and only isoform I reacted with some ACA-positive sera. The 2 isoforms are identical in the first 102 amino acids, but differ greatly in their remaining sequences. Thus, the epitopes responsible for the autoimmunity of CENP-M must reside in the last 72 amino acids of isoform I.

Using a CENP-focused protein microarray, $5 \mathrm{CENP}$ were identified as novel candidate ACA targets in SSc. Of them, CENP-P and -Q showed high sensitivities in ACA-positive sera. Subsequent validation analysis by ELISA and Western blot confirmed that both are ACA autoantigens. Additionally, anti-CENP-P autoantibodies were present in ACA-negative SSc sera at a lower rate. The presence of either anti-CENP-P or anti-CENP-Q was associated with some clinical manifestations and laboratory features.

\section{ACKNOWLEDGMENT}

The authors thank Eric Cox from HiT Center of Johns Hopkins University School of Medicine for text editing, and Haipan Zeng from Beijing Protein Innovation Ltd. Co. for technical assistance.

\section{REFERENCES}

1. Fritzler MJ, Kinsella TD. The CREST syndrome: A distinct serologic entity with anticentromere antibodies. Am J Med 1980;69:520-6.

2. Neil JM. 20 - Centromere autoantibodies. In: Shoenfeld Y, Gershwin ME, Meroni P-L, editors. Autoantibodies. 2nd ed. Burlington, VT: Elsevier; 2007:151-6.

3. Kampolis C, Plastiras S, Vlachoyiannopoulos P, Moyssakis I, Tzelepis G. The presence of anti-centromere antibodies may predict progression of estimated pulmonary arterial systolic pressure in systemic sclerosis. Scand J Rheumatol 2008;37:278-83.

4. Koenig M, Dieude M, Senecal JL. Predictive value of antinuclear autoantibodies: The lessons of the systemic sclerosis autoantibodies. Autoimmun Rev 2008;7:588-93.

5. Muller CD, Paiva ED, Azevedo VF, Radominski SC, Lima Filho $\mathrm{JH}$. Autoantibody profile and clinical correlation in a group of patients with systemic sclerosis in southern Brazil. Rev Bras Reumatol 2011;51:319-24.

6. Nakamura M, Kondo H, Mori T, Komori A, Matsuyama M, Ito M, et al. Anti-gp210 and anti-centromere antibodies are different risk factors for the progression of primary biliary cirrhosis. Hepatology 2007;45:118-27.

7. Granito A, Muratori P, Muratori L, Pappas G, Cassani F, Worthington J, et al. Antibodies to SS-A/Ro-52kD and centromere in autoimmune liver disease: A clue to diagnosis and prognosis of primary biliary cirrhosis. Aliment Pharmacol Ther 2007;26:831-8.

8. Agmon-Levin N, Shapira Y, Selmi C, Barzilai O, Ram M, Szyper-Kravitz M, et al. A comprehensive evaluation of serum autoantibodies in primary biliary cirrhosis. J Autoimmun 
2010;34:55-8

9. Bournia VK, Diamanti KD, Vlachoyiannopoulos PG, Moutsopoulos HM. Anticentromere antibody positive Sjogren's syndrome: A retrospective descriptive analysis. Arthritis Res Ther 2010;12:R47.

10. Nakano M, Ohuchi Y, Hasegawa H, Kuroda T, Ito S, Gejyo F. Clinical significance of anticentromere antibodies in patients with systemic lupus erythematosus. J Rheumatol 2000;27:1403-7.

11. Russo K, Hoch S, Dima C, Varga J, Teodorescu M. Circulating anticentromere CENP-A and CENP-B antibodies in patients with diffuse and limited systemic sclerosis, systemic lupus erythematosus, and rheumatoid arthritis. J Rheumatol 2000; 27:142-8.

12. Respaldiza N, Wichmann I, Ocana C, Garcia-Hernandez FJ, Castillo MJ, Magarino MI, et al. Anti-centromere antibodies in patients with systemic lupus erythematosus. Scand J Rheumatol 2006;35:290-4

13. Atalay C, Dogan L, Atalay G. Anti-CENP-B antibodies are associated with prolonged survival in breast cancer. Future Oncol 2010;6:471-7.

14. Kingwell B, Rattner JB. Mammalian kinetochore/centromere composition: A $50 \mathrm{kDa}$ antigen is present in the mammalian kinetochore/centromere. Chromosoma 1987;95:403-7.

15. Ford AL, Kurien BT, Harley JB, Scofield RH. Anti-centromere autoantibody in a patient evolving from a lupus/Sjogren's overlap to the CREST variant of scleroderma. J Rheumatol 1998; 25:1419-24

16. Rattner JB, Rees J, Arnett FC, Reveille JD, Goldstein R, Fritzler MJ. The centromere kinesin-like protein, CENP-E. An autoantigen in systemic sclerosis. Arthritis Rheum 1996;39:1355-61.

17. Saito A, Muro Y, Sugiura K, Ikeno M, Yoda K, Tomita Y. CENP-O, a protein localized at the centromere throughout the cell cycle, is a novel target antigen in systemic sclerosis. J Rheumatol 2009;36:781-6.

18. Rattner JB, Rao A, Fritzler MJ, Valencia DW, Yen TJ. CENP-F is a .ca $400 \mathrm{kDa}$ kinetochore protein that exhibits a cell-cycle dependent localization. Cell Motil Cytoskeleton 1993;26:214-26.

19. He D, Zeng C, Woods K, Zhong L, Turner D, Busch RK, et al. CENP-G: A new centromeric protein that is associated with the alpha-1 satellite DNA subfamily. Chromosoma 1998;107:189-97.

20. Hsu TC, Chang CH, Lin MC, Liu ST, Yen TJ, Tsay GJ. Anti-CENP-H antibodies in patients with Sjogren's syndrome. Rheumatol Int 2006;26:298-303.

21. Hamdouch K, Rodriguez C, Perez-Venegas J, Rodriguez I, Astola A, Ortiz M, et al. Anti-CENPI autoantibodies in scleroderma patients with features of autoimmune liver diseases. Clin Chim Acta 2011;412:2267-71.

22. Saito A, Muro Y, Sugiura K, Akiyama M. Low prevalence of autoantibodies to CENP-H, -I, -K, -L, -M, -N, -T and -U in a Japanese cohort of anti-centromere positive samples. Immunopharmacol Immunotoxicol 2013;35:57-63 .

23. Volpe A, Ruzzenente O, Caramaschi P, Pieropan S, Tinazzi I, Carletto A, et al. Clinical associations of anti-CENP-B and anti-Sc170 antibody levels measured by multiplexed fluorescent microsphere immunoassay in systemic sclerosis. Rheumatol Int 2009;29:1073-9.

24. Gelber AC, Pillemer SR, Baum BJ, Wigley FM, Hummers LK, Morris S, et al. Distinct recognition of antibodies to centromere proteins in primary Sjogren's syndrome compared with limited scleroderma. Ann Rheum Dis 2006;65:1028-32.

25. Okada M, Cheeseman IM, Hori T, Okawa K, McLeod IX, Yates JR 3rd, et al. The CENP-H-I complex is required for the efficient incorporation of newly synthesized CENP-A into centromeres. Nat Cell Biol 2006;8:446-57.

26. Preliminary criteria for the classification of systemic sclerosis (scleroderma). Subcommittee for scleroderma criteria of the
American Rheumatism Association Diagnostic and Therapeutic Criteria Committee. Arthritis Rheum 1980;23:581-90.

27. LeRoy EC, Black C, Fleischmajer R, Jablonska S, Krieg T, Medsger TA Jr, et al. Scleroderma (systemic sclerosis): Classification, subsets and pathogenesis. J Rheumatol 1988; 15:202-5

28. Heathcote EJ. Management of primary biliary cirrhosis. The American Association for the Study of Liver Diseases practice guidelines. Hepatology 2000;31:1005-13.

29. Vitali C, Bombardieri S, Jonsson R, Moutsopoulos HM, Alexander EL, Carsons SE, et al. Classification criteria for Sjogren's syndrome: A revised version of the European criteria proposed by the American-European Consensus Group. Ann Rheum Dis 2002;61:554-8

30. Hochberg MC. Updating the American College of Rheumatology revised criteria for the classification of systemic lupus erythematosus. Arthritis Rheum 1997;40:1725.

31. Clegg DO, Ward JR. Diagnostic criteria in rheumatoid arthritis. Scand J Rheumatol Suppl 1987;65:3-11.

32. Walker UA, Tyndall A, Czirjak L, Denton C, Farge-Bancel D, Kowal-Bielecka O, et al. Clinical risk assessment of organ manifestations in systemic sclerosis: A report from the EULAR Scleroderma Trials and Research Group database. Ann Rheum Dis 2007;66:754-63.

33. Zhu H, Bilgin M, Bangham R, Hall D, Casamayor A, Bertone P, et al. Global analysis of protein activities using proteome chips. Science 2001;293:2101-5.

34. Hu CJ, Song G, Huang W, Liu GZ, Deng CW, Zeng HP, et al. Identification of new autoantigens for primary biliary cirrhosis using human proteome microarrays. Mol Cell Proteomics 2012;11:669-80

35. Zhu H, Hu S, Jona G, Zhu X, Kreiswirth N, Willey BM, et al. Severe acute respiratory syndrome diagnostics using a coronavirus protein microarray. Proc Natl Acad Sci USA 2006;103:4011-6.

36. Quintana FJ, Farez MF, Viglietta V, Iglesias AH, Merbl Y, Izquierdo $\mathrm{G}$, et al. Antigen microarrays identify unique serum autoantibody signatures in clinical and pathologic subtypes of multiple sclerosis Proc Natl Acad Sci USA 2008;105:18889-94.

37. Fritzler MJ, Rattner JB, Luft LM, Edworthy SM, Casiano CA, Peebles $\mathrm{C}$, et al. Historical perspectives on the discovery and elucidation of autoantibodies to centromere proteins (CENP) and the emerging importance of antibodies to CENP-F. Autoimmun Rev 2011;10:194-200

38. Rodriguez-Reyna TS, Hinojosa-Azaola A, Martinez-Reyes C, Nunez-Alvarez CA, Torrico-Lavayen R, Garcia-Hernandez JL, et al. Distinctive autoantibody profile in Mexican Mestizo systemic sclerosis patients. Autoimmunity 2011;44:576-84.

39. McNeilage LJ, Youngchaiyud U, Whittingham S. Racial differences in antinuclear antibody patterns and clinical manifestations of scleroderma. Arthritis Rheum 1989;32:54-60.

40. Pakunpanya K, Verasertniyom O, Vanichapuntu M, Pisitkun P, Totemchokchyakarn K, Nantiruj K, et al. Incidence and clinical correlation of anticentromere antibody in Thai patients. Clin Rheumatol 2006;25:325-8.

41. Tager RE, Tikly M. Clinical and laboratory manifestations of systemic sclerosis (scleroderma) in Black South Africans. Rheumatology 1999;38:397-400.

42. Steen VD. Autoantibodies in systemic sclerosis. Semin Arthritis Rheum 2005;35:35-42.

43. Jacobsen S, Halberg P, Ullman S, Van Venrooij WJ, Hoier-Madsen $\mathrm{M}$, Wiik A, et al. Clinical features and serum antinuclear antibodies in 230 Danish patients with systemic sclerosis. Br J Rheumatol 1998;37:39-45.

44. Mayes MD, Lacey JV Jr, Beebe-Dimmer J, Gillespie BW, Cooper B, Laing TJ, et al. Prevalence, incidence, survival, and disease characteristics of systemic sclerosis in a large US population. Arthritis Rheum 2003;48:2246-55. 